Sinergias educativas

ISSN: 2661-6661

compasacademico@icloud.com

Grupo Compás

Ecuador

\title{
La actividad fi\#sica como mecanismo de formacion integral del ser humano
}

\author{
Casquete Fabre, Mari\#a Fernanda \\ La actividad fi\#sica como mecanismo de formacion integral del ser humano \\ Sinergias educativas, vol. 2, núm. 1, 2017 \\ Grupo Compás, Ecuador \\ Disponible en: http://www.redalyc.org/articulo.oa?id=573561688004
}

Esta obra está bajo una Licencia Creative Commons Atribución-NoComercial-SinDerivar 4.0 Internacional. 


\section{La actividad fi\# sica como mecanismo de formacion integral del ser humano}

Sinergias educativas, vol. 2, núm. 1, 2017

Grupo Compás, Ecuador

Recepción: 15 Mayo 2016

Aprobación: 25 Noviembre 2016

Redalyc: http://www.redalyc.org/ articulo.oa?id $=573561688004$

\author{
Physical activity as a mechanism of integral formation of the \\ human being \\ María Fernanda Casquete Fabre casquetefabre@Hotmail.com \\ Unidad Educativa Nueva Semilla, Ecuador \\ http://orcid.org/0000-0004-0219-587231
}

Resumen: Dentro del complejo mundo de ser humano vemos que es de vital importancia para su formacio\#n integral la actividad fi\#sica sea cual sea el modo de realizarlo ya que va desde una caminata hasta una competencia de atletismo o una marato\#n. Los ejercicios ayudan a la prevencio\#n de enfermedades cro\#nicas asi\# mismo destaca su importancia en los tratamientos de drogodependientes y/o cualquier tipo de enfermedad. Para evitar no solo dolencias fi\#sicas y alteraciones psicolo\#gicas es muy importante introducir desde temprana edad la actividad fi\#sica para que se constituya en un ha\#bito hasta llegar a la madurez absoluta. Cambiando su modo de ver la realidad de su cuerpo y como cambia su estado ani\#mico sin darse cuenta que al ejecuta cualquier tipo de movimiento desarrolla todo su potencial y da lo mejor de si\# en el medio donde se desenvuelve sea este casa o trabajo o simplemente mejora sus relaciones personales.

Palabras clave: Personales, humano, drogadicto.

Abstract: Within the complex world of being human we see that physical activity is of vital importance for its integral formation whatever the way of doing it since it goes from a walk to an athletic competition or a marathon. The exercises help the prevention of chronic diseases and also highlights its importance in the treatment of drug addicts and / or any type of disease.

To avoid not only physical ailments and psychological alterations, it is very important to introduce physical activity from an early age so that it becomes a habit until it reaches absolute maturity. Changing the way you see the reality of your body and how your mood changes without realizing that when you execute any type of movement, you develop your full potential and do your best in the environment where this house or work takes place or simply improves your personal relationships

Keywords: Personal, human, drug addict.

\section{INTRODUCCIÓN}

El ser humano es una entidad global formada por tres dimensiones, biológica, psicológica y social, que dan lugar a manifestaciones biosociales, psicobiológicas y psicosociales del desarrollo. Como soporte y puente entre la persona y el ambiente en el que e\#sta se desenvuelve, y tambie\#n como conexión entre muchas de las operaciones humanas entre sí, se encuentra la motricidad (actividad física, deporte), la cual repercute en tres grandes a\#reas: biomotriz, psicomotriz y sociomotriz. Por tanto, la pra\#ctica deportiva puede hacerse partícipe de la formación integral del ser humano puesto que guarda relación con todos estos elementos, no limita\#ndose u\#nicamente a las repercusiones físicas, sino teniendo tambie\#n una gran capacidad de influencia sobre las funciones 
psicológicas (emocionales) y sociales (relacionales). (GUTIE\#RREZ SANMARTÍN, 2004)

Introducir ha\#bitos saludables desde edades tempranas es indispensable para reducir el riesgo de enfermedades cro\#nicas, incidiendo en el correcto desarrollo del joven trascendiendo tambie\#n en la duracio\#n y calidad de vida del ser humano. La actividad fi\#sica es una de las variables que ma\#s influyen sobre la salud.

Debido a muchas razones dependientes del entorno del nin\#o, joven o adulto existen situaciones de sedentarismo o de escasa actividad fi\#sica, influye de manera positiva la educacio\#n fi\#sica en el ambiente escolar como un a\#trea aprovechable para el incremento general de la actividad fi\#sica al mejorar los ha\#bitos de vida, las relaciones socio-afectivas llegando a influir en el rendimiento cognitivo; todos estos elementos son indicadores importantes de la salud.

\section{MATERIALES Y MÉTODOS}

La actividad fi\#sica es considerada un elemento integrador ya que ensen\#a a ser responsables, ayuda a la prevencio\#n y tratamiento de drogodependencias incluso sirve como medio de reinsercio\#n en las instituciones penitenciarias.

Hace ya ma\#s de una de\#cada que Oja y Telama (1991), recogiendo las conclusiones de los trabajos presentados en el Congreso Mundial de Deporte para Todos, celebrado en Finlandia en 1990, hacen especial hincapie\# en el valor del deporte para la formación integral de la persona, es decir, para todas y cada una de sus a\#reas del desarrollo, tanto para las físicas (forma física, salud y prevención de la enfermedad) como para las psicológicas (autoestima, personalidad, calidad de vida) y las sociales (relación con los dema\#s, rendimiento en el empleo adema\#s de poder influir sobre el desarrollo moral y la promoción de diversos valores sociales y personales

Dentro de nuestra sociedad el deporte cada vez se incorpora ma\#s y es considerado un feno\#meno social que de gran manera mejora la calidad de vida del ser humano, el cual esta\# acostumbrado generalmente al ocio actuando como un catalizador en la salud fi\#sica y psicolo\#gica de la persona y no solo eso sino cada vez ma\#s se lo usa como producto, objeto de intercambio social y eje econo\#mico en muchos casos.

La actividad fi\#sica nos es inmensamente u\#til para combatir las enfermedades de moda con alto nivel de mortalidad, como son las cardiovasculares, en cuya etiologi\#a se encuentra el sedentarismo y el desequilibrio, tensio\#n fi\#sica-inaccio\#n fi\#sica como factores de alta responsabilidad. Pero podemos destacar que son terapias no farmacolo\#gicas que actu\# an de manera efectiva para reducir el estre\#s ya sea e\#ste provocado por el trabajo o situaciones de casa y/o sentimentales, ayuda en los trastornos de suen\#o, depresio\#n, ansiedad, etc., que son normales a medida que vamos envejeciendo incluso ya en la edad madura la pra\#tctica continua otorga grandes beneficios a la salud mental de la misma. 
En e\#sta e\#poca donde el trabajo y/o ocupaciones del hogar demandan mucho tiempo para su ejecucio\#n; el tiempo libre que se tiene se ha convertido en u\#nica vi\#a para que el individuo se desarrolle social y personalmente con esto no podemos decir que se dedican so\#lo a relaciones personales sino tambie\#n a hacer algu\#n tipo de actividad fi\#sica sea la pra\#ctica de un deporte, baile, ir a un gimnasio, correr entre tantas cosas que con el simple hecho de mover el cuerpo realizamos ejercicio que nos ayuda de manera directa a cualquier situacio\#n buena o mala por la que se atraviesa.

Desde el a\#mbito de las ciencias del deporte, Diem (1973), Cagigal (1957) y Laín Entralgo (1991) ensalzan el aspecto humano del deporte que, por su total implicación corporal, ritual y espiritual, esta\# llamado a desempen\#ar cada vez ma\#s un profundo rol de garantía humanística. El deporte se hace así sinónimo de conducta humana puesto que ofrece una respuesta mu\#ltiple para abordar los modos de interpretar la vida. Los teóricos que estudian el deporte bajo el prisma de la orientación filosófica persiguen comprender la realidad para relacionarlo con el ser y el conocimiento. Heidegger (2003) bajo el prisma del existencialismo, profundiza en la resistencia como fórmula de vida. La resistencia (el trabajo, el esfuerzo) es la variante que necesita el ser en el mundo para mantenerse vivo. $\mathrm{Y}$ es que la resistencia ha sido un concepto fecundo en las tareas de toda expresión y creatividad del hombre en el deporte, y no sólo como capacidad ba\#sica. Pues bien, el concepto cla\#sico de entrenamiento, que viene de askesis (ascetismo) se refería a la doctrina y actitud que busca la perfección del hombre mediante la pra\#ctica de una vida austera, mística y mortificante, al igual que muchas tradiciones religiosas. No es casualidad que una civilización tan interesada en el deporte como la griega prestara atención a la realidad del placer y del dolor.

El deportista siente que cuando el dolor se extingue se experimenta una intensa sensacio\#n de placer. Desde la psicologi\#a utilizan la expresio\#n de experiencia cumbre (Peak Experience); o adiccio\#n positiva (BUENO; CAPDEVILLA; (Cruz, 2013), HERNA\#NDEZ). (Cruz, 2013)

La actividad fi\#sica, movimiento y/o deporte como ente regulador del buen funcionamiento del cuerpo humano y su correcta formacio\#n dejamos en este arti\#culo plasmado la gran importancia que esto genera en el ser humano.

\section{CONCLUSIONES}

La actividad fi\#sica es un factor determinante de la salud relacionado con el estilo de vida y su pra\#ctica regular esta\# asociada con disminucio\#n de enfermedades cro\#nicas, es por esto la vital importancia de la pra\#ctica de la misma para la correcta formacio\#n integral de la persona sin importar raza, condicio\#n social, limitaciones fi\#sicas, creencias religiosas ni ge\#nero. Mejoramos la calidad de vida no solo nuestra sino de los que nos rodean ya que siendo ejemplo y mostrando capacidades podemos contagiar al realizar ejercicios o cualquier actividad que incluya movimiento para la correcta formacio\#n. Cabe recalcar 
que la alimentacio\#n y la correcta hidratacio\#n son tambie\#n parte fundamental del buen desarrollo del ser humano no dejando atra\#s tambie\#n la parte psicolo\#gica en donde el deporte o actividad actu\#a de manera extraordinaria.

El objetivo principal de e\#ste arti\#culo es dar a conocer la importancia de movernos, saber hasta do\#nde podemos llegar si nos proponemos y nos desafiamos; saber de lo que somos capaces de realizar y co\#mo hacerlo de manera efectiva. Con la ayuda de una buena nutricio\#n acorde a las necesidades que se nos vayan presentando. Pronto descubriremos que al realizar una pra\#tcica efectiva de un ejercicio o una simple caminata otorgaremos a nuestro cuerpo beneficios innumerables y descubriremos realmente lo que somos capaces de demostrar y sobretodo con una correcta gui\# a avanzaremos hacia el nivel deseado.

\section{Referencias}

Área de Actividad Física y Salud (2008). Área de Actividad Física y Salud. Cultura, ciencia y deporte. 3(8). 69-102.

Cachán Cruz, R. (2013). El deporte, proyeccio\#n, espejo y si\#mbolo cultural: reflexio\#n sobre los deportes de sacrificio y su transmisión de valores en el contexto socioeducativo. Movimento. 19(3), 315, 336.

GUTIE\#RREZ SANMARTI\#N, M. (2004). EL VALOR DEL DEPORTE EN LA EDUCACIO\#N INTEGRAL. Revista de Educacio\#n, 335 (1),105- 126. doi:http://www.revistaeducacion. mec.es/re335/ re335_10.pdf

Rangel Caballero, L. Z. (2015). Actividad fi\#sica y composicio\#n corporal de estudiantes universitarios de cultura fi\#sica, deporte y recreacio\#n. Rev Univ Ind Santander Salud. Revista de la Universidad Industrial de Santander. Salud, 47(3), 281-290. doi: http://dx.doi.org/10.18273/ revsal. v47n3-2015004 